\title{
Bioavailability and antioxidant capacity of plant extracts rich in polyphenols, given as a single acute dose, in sheep made highly susceptible to lipoperoxidation
}

\author{
Cécile Gladine ${ }^{1}$, E. Rock ${ }^{2}$, C. Morand ${ }^{2}$, D. Bauchart ${ }^{1}$ and D. Durand ${ }^{1 *}$ \\ ${ }^{1}$ INRA, Research Unit on Herbivores, Nutrients and Metabolisms Group, 63122 Saint-Genès-Champanelle, France \\ ${ }^{2}$ INRA, Research Unit on Human Nutrition, Metabolic Stress and Micronutrient group, 63122 Saint-Genès-Champanelle, France
}

(Received 26 September 2006 - Revised 7 February 2007 - Accepted 2 March 2007)

Plant extracts rich in polyphenols (PERP) could represent interesting alternative antioxidants but their use in ruminants needs further investigation since the antioxidant capacity of PERP could be altered by digestive processes. The aim of the study was to investigate the bioavailability and the antioxidant capacity of four PERP (rosemary; grape; citrus; marigold) in ruminants made highly susceptible to lipoperoxidation by a continuous linseed oil infusion ( $4 \% \mathrm{DM})$ in the duodenum. The PERP were given, as a single acute dose (10\% DM), directly into the rumen of sheep ( $n$ 5) and blood was then collected every $3 \mathrm{~h}$ over a period of $30 \mathrm{~h}$. Grape was particularly efficient to enhance the plasma total antioxidant status $(P<0.05)$. Moreover, many new polyphenols were detected in the plasma and the identification of epicatechin in the grape group suggested that, contrary to monogastrics, ruminants can benefit from the antioxidant effect of polymeric proanthocyanidins. Finally, the four PERP tested, and more especially marigold, significantly reduced plasma susceptibility to liperoxidation (mean increase of lag phase: +5.9 min, $P<0.02$; mean reduction of oxidation rate: $-1.7 \mathrm{~A}_{234} / \mathrm{min}, P<0.01$ ). In conclusion, the digestive processes in ruminants do not inhibit the antioxidant properties of PERP in vivo and are beneficial by improving the biological effect of polymeric proanthocyanidins. Further experiments are now necessary to determine the optimum dose of administration and to characterize the bioactive molecules.

Polyphenols: Bioavailability: Antioxidant: Ruminant: $n-3$ PUFA

Domestic animals, just like man, are frequently exposed to oxidative stress $^{1}$, involving an overproduction of reactive oxygen species that cause oxidative damage to all macromolecules within the cell ${ }^{2}$. In domestic animals, oxidative stress has been associated with the deterioration of many physiological functions including growth and reproduction as well as immunity ${ }^{3}$. The oxidative deterioration of lipids (or lipoperoxidation) is particularly involved in this phenomenon since lipids are the macromolecules that are the most susceptible to peroxidative processes, especially when they are rich in PUFA $^{4}$

Dietary fat supplementation was proposed 40 years ago for ruminants in order to increase, at a low cost, the energetic density of diets ${ }^{5}$. This strategy was improved by the use of vegetal sources rich in PUFA from the $n-3$ family (n-3 PUFA). Indeed, these fatty acids are believed to be beneficial for human health and by giving them to domestic animals it is possible to increase the content of $n-3$ PUFA in animal products and therefore to improve their nutritional value ${ }^{6,7}$. However, since $n$-3 PUFA are highly susceptible to peroxidation both in plasma and tissues 8,9 , it appeared that a supply of antioxidants should be recommended to preserve the health of animals and the oxidative stability of their products ${ }^{6}$.
Vitamin E is a synthetic antioxidant commonly used in animal nutrition and the bioefficiency of vitamin E supplement to reduce lipoperoxidation in ruminant products has been largely demonstrated ${ }^{6}$. However, its ability to limit lipoperoxidation is not sufficient when $n-3$ PUFA intake is increased $^{10,11}$. This could be due to its restricted site and mechanism of action but it has also been reported that supplementation with vitamin $\mathrm{E}$ is efficient until a certain threshold, beyond which vitamin $\mathrm{E}$ ingested is catabolized or eliminated in faeces and urine ${ }^{1}$. Moreover, vitamin $\mathrm{E}$ has many other biological effects, including modulation of gene expression or inhibition of protein kinase $\mathrm{C}^{12}$, and a pro-oxidant action was also observed in vitro ${ }^{13}$. On the basis of these observations, it is likely that other types of antioxidants, given alone or in association with vitamin $\mathrm{E}$, could be more efficient and less deleterious than the use of megadoses of vitamin E alone to improve the antioxidant protection of animals.

Recent search for new antioxidants focused on natural molecules to satisfy consumer concerns over safety and toxicity. Among natural antioxidants that could be used in animal nutrition, we hypothesized that plant extracts rich in polyphenols (PERP) would be good candidates since polyphenols are abundant in many plants and PERP can then be readily produced at

Abbreviations: BS, blood sample; CD, conjugated diene; CE, cholesteryl ester; CI, citrus; FC, free cholesterol; GP, grape; iAUC, incremental area under the curve; MA, marigold; PERP, plant extracts rich in polyphenols; PL, phospholipid; RO, rosemary; TAS, total antioxidant status.

* Corresponding author: Denys Durand, fax +33(0)4 736246 39, email durand@clermont.inra.fr 
low cost. Moreover, in contrast with vitamin E, polyphenols exhibit various antioxidant properties ${ }^{14}$. Actually, PERP, such as herbs or spices, are already used as antioxidant additives in the food industry, suggesting that this natural approach is relevant ${ }^{15,16}$. However, the use of PERP as dietary antioxidants to preserve both the health of animals and the oxidative stability of their products needs further investigations, since studies conducted in rodents and human subjects showed that many polyphenols are poorly absorbed, intensively metabolized or rapidly excreted ${ }^{17}$. Feeding trials conducted in poultry nevertheless reported beneficial effects of oregano extract in muscle tissue $\mathrm{e}^{18-20}$ but the results cannot be directly extrapolated to ruminants because of the bacterial fermentation processes occurring in rumen ${ }^{21}$, which could greatly affect the bioavailability of PERP and subsequently their in vivo antioxidant capacity.

The aim of the present study was to investigate the bioavailability and the antioxidant capacity of PERP in the plasma of ruminants made highly susceptible to lipoperoxidation. For this purpose, four PERP, known for their bioefficiency in rats $^{22,23}$, were given, as a single acute dose, directly into the rumen of sheep. Parameters related to bioavailability and antioxidant capacity of PERP were measured in the plasma collected every $3 \mathrm{~h}$ during a period of $30 \mathrm{~h}$ after the PERP administration.

\section{Materials and methods}

\section{Animal management}

All experiments were conducted in accordance with the national legislation on animal care (certificate authorization to experiment on Living Animal No. 7740, Ministry of Agriculture and Fish Products, Paris, France). Five castrated Texel sheep (18 months old, 50 (SEM 4) kg body weight) were used in a $5 \times 5$ Latin square design. They were surgically fitted with a rumen cannula (polyvinyl chloride; $60 \mathrm{~mm}$ o.d.) and a T-shaped cannula (silicone rubber, $17 \mathrm{~mm} \mathrm{o.d)} \mathrm{in} \mathrm{the} \mathrm{prox-}$ imal duodenum. After the post-surgical period (1 month), the sheep were penned individually into metabolism cages and in controlled temperatures $\left(18\right.$ to $\left.22^{\circ} \mathrm{C}\right)$.

\section{Diets and plant extracts}

Diets distributed to the animals throughout the entire experiment consisted of $840 \mathrm{~g} / \mathrm{d}$ meadow hay and $360 \mathrm{~g} / \mathrm{d}$ concentrate, which was composed of barley (43\% diet DM), beet pulp (40\%), soyabean meal (12\%) and molasses (5\%). Diets were distributed twice per d at 0800 and 1600 hours and feed intake was recorded each day.

Four PERP were selected according to the economical and technical requirements of animal nutrition and were supplied by the Phytosynthèse society (Saint-Bonnet de Rochefort, France). The PERP were obtained from rosemary (RO; Rosemarinus officinalis, entire plant used), grape (GP; Vinis vitifera, peel and seed used, aqueous extraction), citrus (CI; Citrus paradisi, fruit used, aqueous extraction) and marigold (MA; Calendula officinalis, flower used). The content of proanthocyanidins in GP represented more than $80 \%$ of the polyphenols, and polymeric proanthocyanidins represented $70 \%$ of the total proanthocyanidins according to the manufacturer's details. Concerning CI extract, it contained more than $95 \%$ naringin. RO was not purified and was then assumed to contain all the usual polyphenols ${ }^{24}$, i.e. diterpenes phenolic and rosmarinic acid. MA was not purified and was then assumed to contain all the usual phenolic acids ${ }^{25}$, i.e. vanillic, caffeic, p-coumaric and ferulic acids. Moreover, MA was analysed for its carotenoid content and xanthophylls represented $>1 \%$ of plant. In addition to the manufacturer's details, the polyphenol content (Folin-Ciocalteu assay) as well as the in vitro reducing capacity (1,1-diphenyl-2-picrylhydrazyl assay) of the four PERP were determined as previously reported $^{22}$ and are reported in Table 1.

\section{Experimental design}

The experiment was divided into two phases. The first phase (phase 1) lasted $23 \mathrm{~d}$ and consisted of a continuous linseed oil infusion (4\% DM ingested) via the duodenum cannula. This procedure, developed in our laboratory ${ }^{9}$, aimed to protect $n-3$ PUFA from rumen biohydrogenation and to increase the $n$-3 PUFA content in plasma, making it highly susceptible to lipoperoxidation. During the second phase (phase 2, 22 d), animals were still continuously infused with linseed oil but they were also given one of the four PERP or chopped hay (control treatment), according to a $5 \times 5$ Latin square design (i.e. $5 \mathrm{~d}$ of administration and five sheep). Sub-periods lasted $3 \mathrm{~d}$ and the administration was performed before the morning meal. It consisted of one acute dose (10\% DM intake) administrated via the rumen cannula. The use of the rumen cannula aimed to control the time of administration and the amount of PERP administrated.

\section{Blood sampling}

A set of four blood samples (BS; $10 \mathrm{ml}$ ) was collected by venipuncture from the jugular vein during phase 1 to determine the effect of linseed oil infusion on lipaemia. A first BS was collected before the beginning of the linseed oil infusion (time zero, $\mathrm{T}_{0}$ ) and the other three were collected 9,16 and $23 \mathrm{~d}$ after the beginning of the infusion $\left(\mathrm{T}_{9}, \mathrm{~T}_{16}, \mathrm{~T}_{23}\right)$.

During phase 2, BS $(20 \mathrm{ml})$ were collected before and after the PERP administration (Fig. 1) as follows: two samples (BS1 and 2) were respectively collected $60 \mathrm{~min}$ and $15 \mathrm{~min}$ before the PERP administration in the rumen and were designated as reference samples (REF samples). After the PERP administration, BS were collected every $3 \mathrm{~h}$ for $30 \mathrm{~h}$ (BS3 to BS12). Time of sampling was determined by taking into account the retention times of the liquid and the solid

Table 1. Polyphenol content and reducing potential of plant extracts obtained from rosemary (RO), grape (GP), citrus $(\mathrm{Cl})$ or marigold (MA)

\begin{tabular}{lrrrr}
\hline & \multicolumn{4}{c}{ Plant extracts } \\
\cline { 2 - 5 } & RO & GP & Cl & MA \\
\hline Polyphenol content* & 16 & 651 & 213 & 77 \\
Reducing potential† & 469 & 6630 & 212 & 962 \\
\hline *Gallic acid equivalent, mg/g DM. & & \\
†Trolox equivalent antioxidant capacity, $\mu \mathrm{mol} / \mathrm{g}$ & DM.
\end{tabular}




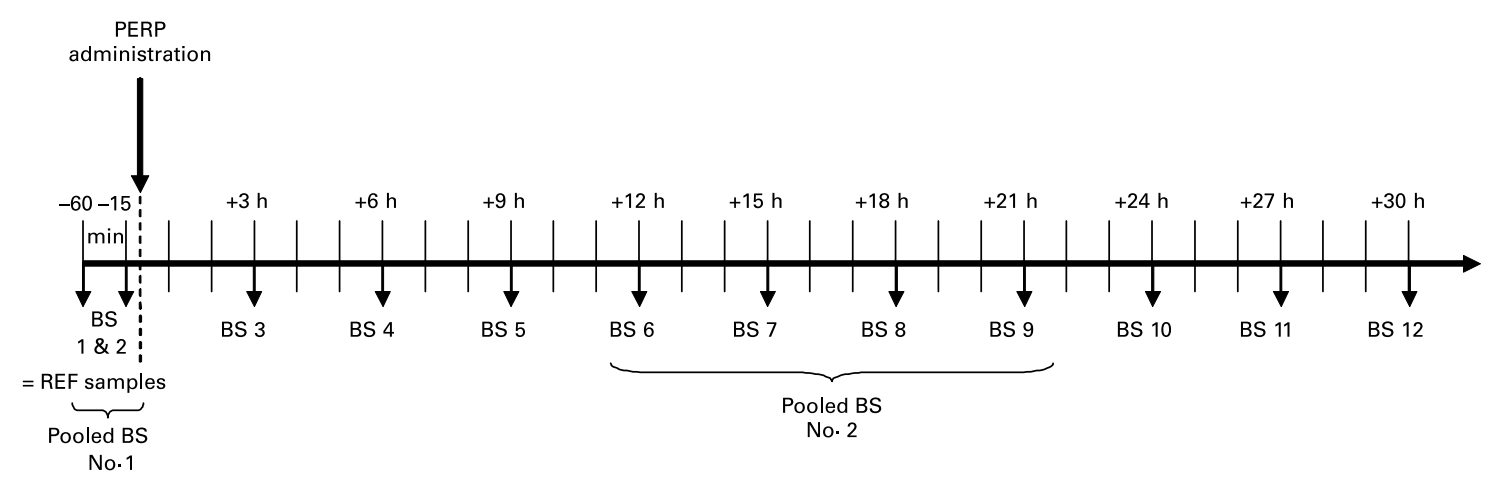

Fig. 1. Scheme of the blood sampling procedure after plant extracts rich in polyphenol (PERP) administration in the rumen of sheep ( $n$ 5). Blood sample (BS) 1 and 2 were collected before PERP administration and designated as reference samples (REF samples). PERP (rosemary (RO), grape (GP), citrus (Cl) and mari gold (MA)) or chopped hay (control) were put, as a single acute dose (10\% DM ingested), directly in the rumen of sheep ( $n 5)$ according to a $5 \times 5$ Latin square design. Pooled blood samples No. 1 and No. 2 constituted for the analysis of the plasma polyphenol patterns and the monitoring of conjugated diene generation.

phases of the rumen content, since polyphenols could be eluted with both phases. The sampling procedure was used for each of the five sheep and was repeated for each of the $5 \mathrm{~d}$ of administration.

BS were collected into sterile vacutainer tubes containing: 1) $\mathrm{Na}_{2}$-EDTA $(3 \mathrm{~mm})$ for the determination of the lipid classes (TAG; phospholipid (PL); free cholesterol (FC); cholesteryl ester (CE); NEFA) and for polyphenol analysis; 2) lithium heparin $(5 \mathrm{UI} / \mathrm{ml})$ for total antioxidant status (TAS) measurement; 3) sodium citrate ( $18 \mathrm{~mm})$ to monitor the kinetics of conjugated diene (CD) generation. Plasma was separated from blood by centrifugation $\left(1600 \mathrm{~g}, 10 \mathrm{~min}\right.$ at $\left.4^{\circ} \mathrm{C}\right)$. The plasma collected for the analysis of polyphenols was immediately acidified to $\mathrm{pH} 6.8$ with $10 \mathrm{mmol} / \mathrm{l}$ acetic acid. All plasmas were aliquoted and stored at $-80^{\circ} \mathrm{C}$ until analysis

\section{Blood sample analysis}

Plasma lipids. Concentrations of the major lipid classes were determined in BS collected during the first step of the experimental period. The enzymatic method used to determine TAG content ${ }^{26}$ required the Kit PAP 150 (ref. RC 1260-30) supplied by BioDirect (Charbonnières-les Bains, France). PL were determined by the enzymatic method of Trinder using the BioDirect Kit (ref. RC 1254-01; BioDirect). CE and FC were respectively determined enzymatically with the reagent Kit Cholesterol Liquide and Cholesterol Libre Enz. Color supplied by BioMérieux (Chennevièvre-les-Louvres, France). Plasma NEFA were determined enzymatically with the Wako Kit (Unipath, Dardilly, France). Total lipids corresponded to the sum of TAG + PL + FC + CE + NEFA.

Polyphenol pattern determination. This analysis was performed in plasma collected during phase 2 and pooled as follows: a first pooled sample (pooled BS No. 1) was constituted by mixing the two REF samples collected before the PERP administration (BS1 and 2, see Fig. 1), a second pooled sample (pooled BS No. 2) consisted of the mixture of the samples collected 12, 15, 18 and $21 \mathrm{~h}$ after PERP administration in the rumen (BS6, BS7, BS8, BS9, see Fig. 1). This period of time corresponded to the period during which TAS reached the maximal value for most experimental groups (see Fig. 2). For each day of PERP administration ( $n$ 5), two pooled samples (BS1 and BS2) were then constituted for each sheep ( $n$ 5). At the end of the experimental period, we finally pooled all together the BS1 samples from sheep given the same PERP (five final pooled samples constituted) and the BS2 samples from sheep given the same PERP (five final pooled samples constituted).

These two pooled samples were prepared for each extract (n 5) and for each day of administration ( $n$ 5). Finally, we pooled the five pooled BS No. 1 and the five pooled BS No. 2 obtained from the five sheep receiving the same extract, leading to ten final pooled samples, which were used for polyphenol pattern analysis.

For the polyphenol analysis, plasma samples $(180 \mu \mathrm{l})$ were acidified to $\mathrm{pH} 4.9$ with $20 \mu \mathrm{l} 0.58 \mathrm{~mol} / \mathrm{l}$ acetic acid and were incubated at $37^{\circ} \mathrm{C}$ with a mixture of $\beta$-glucuronidase $(1000$ units) and sulfatase (100 units) from Helix pomatia (Sigma, Chesnes, France). This enzymatic procedure hydrolysed the circulating form of polyphenols (glucuronides and sulphate) to release the aglycone form. Incubation times were selected according to the main polyphenols known to be present in each extract ${ }^{27}$ : RO (rosmarinic acid), GP (proanthocyanidins), CI (naringin) or MA (phenolic acids) and were set as follows: 30 min for plasma collected after GP administration; $4 \mathrm{~h}$ for plasma collected after RO and MA administration; $5 \mathrm{~h}$ for plasma collected after CI administration. The aglycones released were then extracted with $500 \mu \mathrm{l}$ methanol/HCl $200 \mathrm{mmol} / \mathrm{l}$ and centrifuged at $14000 \mathrm{~g}$ for $4 \mathrm{~min}$ at $4^{\circ} \mathrm{C}$. Polyphenols in the resulting supernatant fractions were analysed by HPLC 8-electrodes-CoulArray Model 5600 system (Eurosep, Cergy, France) fitted with a $150 \times 4.6$ Hypersil BDS C18$5 \mu \mathrm{m}$ column (Interchim, Montluçon, France). The mobile phases consisted of $5 \%$ acetonitrile in $30 \mathrm{mmol} / 1 \mathrm{NaH}_{2} \mathrm{PO}_{4}$ $\mathrm{pH} 3.0$ for solvent $\mathrm{A}$ and $50 \%$ acetonitrile in $30 \mathrm{mmol} / \mathrm{l}$ $\mathrm{NaH}_{2} \mathrm{PO}_{4} \mathrm{pH} 3.0$ for solvent $\mathrm{B}$. The analysis was performed using a gradient elution procedure as follows: flow rates $0.80 \mathrm{ml} / \mathrm{min} ; 0-3 \mathrm{~min}$ : solvent A $100 \% ; 3-73 \mathrm{~min}$ : from solvent A $100 \%$ to solvent B $100 \%$; 73-76 min: solvent B $100 \%$; 76-95 min solvent A $100 \%$. For detection, electrode potentials were set at $0,120,240,360,480,600,720$ and $840 \mathrm{mV}$. These chromatographic and electrochemical conditions allow the separation of both phenolic acids and flavonoids. The antioxidant capacity of the polyphenols was relative to their electrochemical behaviour, the molecules detected on electrodes set at low potentials (from 0 to 


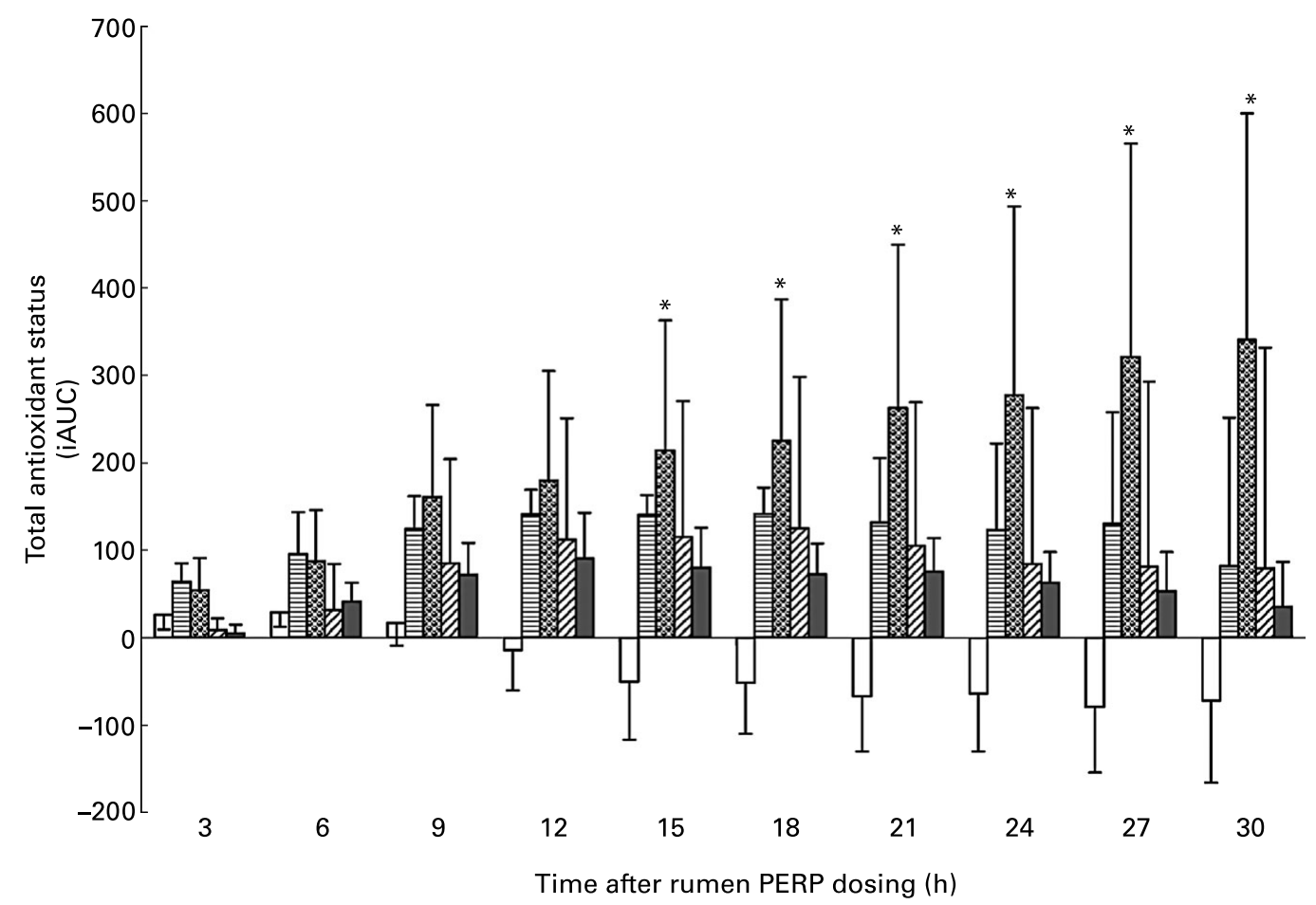

Fig. 2. Evolution with time (from 3 to $30 \mathrm{~h}$ ) of plasma total antioxidant status (expressed as incremental area under the curve (iAUC) calculated by using the linear trapezoidal rule) of sheep $(n 5)$ given, directly in the rumen, as a single acute dose (10\% DM ingested), one of the four plant extracts rich in polyphenols (PERP): $\square$, control; $\square$, rosemary; grape; $\boldsymbol{\square}$, citrus; $\mathbf{\square}$, marigold. Values are means with their standard errors for five sheep. Experimental values were significantly different from the control value: ${ }^{*} P<0.05$. For details of diets and procedures, see Materials and Methods.

$480 \mathrm{mV}$ ) being the most antioxidant. Polyphenols in plasma of experimental groups were visualized by comparing with the control group as well as by comparing the chromatographic profiles obtained from plasma collected before and after PERP administration. Moreover, standards of phenolic acids (rosmarinic, chlorogenic, ferulic, isoferulic, caffeic, p-coumaric, vanillic acids) and of flavonoids (proanthocyanidins B1, B2 and B3, catechin, epicatechin, naringenin) were used to identify specific polyphenols potentially present in plasma.

Total antioxidant status. TAS was measured in plasma samples collected before (BS 1 and 2, see Fig. 1) and during (BS3 to 12, see Fig. 1) the $30 \mathrm{~h}$ following the ruminal administration of each PERP. This measurement was performed to determine the contribution of PERP to the oxidative stability of plasma and to provide a first indication of PERP bioavailability. The analytical method was based on the absorbance of the $\mathrm{ABTS}^{\circ+}\left(2,2^{\prime}\right.$-azinobis-3-ethylbenzothiazoline-6-sulphonic acid ${ }^{28}$ cation. Briefly, production of $\mathrm{ABTS}^{\circ+}$ was initiated by addition of $450 \mu \mathrm{m}$-hydrogen peroxide in $1 \mathrm{ml}$ quartz cuvette, containing $12 \mu \mathrm{l}$ plasma, $500 \mu \mathrm{M}$-ABTS and $48.8 \mu \mathrm{M}$-metmyoglobin diluted in degassed PBS (5 mM, pH 7.4). Absorbance at $732 \mathrm{~nm}$ was measured immediately $\left(\mathrm{A}_{\mathrm{t} 0}\right)$ and $3 \mathrm{~min}\left(\mathrm{~A}_{\mathrm{t} 3}\right)$ after the addition of $\mathrm{H}_{2} \mathrm{O}_{2}$ at $37^{\circ} \mathrm{C}$ using a double-beam spectrophotometer (Uvikon 923; Kontron Analysis Division, Zurich, Switzerland). Absorbance of a blank (i.e. containing degassed-PBS instead of plasma) was also determined to calculate the inhibition percentage of the reaction as follows:

$$
\% \text { inhibition }=\frac{\left(A_{t 0}-A_{t 3}\right)_{\text {blank }}-\left(A_{t 0}-A_{t 3}\right) \text { plasma }}{\left(A_{t 0}-A_{t 3}\right)_{\text {blank }}} \times 100
$$

Inhibition percentage was converted into $\mu$ mol of trolox equivalent antioxidant capacity using a trolox standard curve ranging from 0 to $2500 \mu \mathrm{M}$.

Conjugated dienes. Susceptibility of plasma PUFA to peroxidation was determined by measuring the kinetics of $\mathrm{CD}$ generation on plasma collected during Phase 2. The analysis was performed on fifty pooled plasma samples, which corresponded to the pooled BS No. 1 and the pooled BS No. 2 constituted for each sheep $(n$ 5) at each day of administration ( $n$ 5) as described earlier. Oxidation process was initiated by the addition of $\mathrm{CuCl}_{2}(10 \mathrm{~mm})^{29}$. $\mathrm{CD}$ absorbance was continuously monitored at $234 \mathrm{~nm}$ at $37^{\circ} \mathrm{C}$ for $10 \mathrm{~h}$ using the double-beam spectrophotometer (Uvikon 923; Kontron Analysis Division). The kinetics of CD generation were divided into two phases, from which two parameters were calculated as previously described ${ }^{30}$ : 1) the length of lag phase ( $\min )$ corresponding to the resistance time of PUFA against peroxidation; 2) the maximum rate of peroxidation $\left(\mathrm{A}_{234} / \mathrm{min}\right)$, which corresponds to the slope of the curve and represents the speed of the propagation phase.

\section{Statistical analysis and calculation}

All data were subjected to ANOVA analysis by using the general linear model procedure of SAS (SAS Institute, Cary, NC, USA). Significance was declared at $P<0.05$ and $P \leq 0.10$ was considered as a trend. When the effect was declared as a trend or significant, means of the five groups were compared using Student's $t$ test $^{29}$. 
The evolution of TAS over the $30 \mathrm{~h}$ following the plant extract administration was expressed as an incremental area under the curve (iAUC). The initial calculation was done to determine the evolution between the TAS determined before

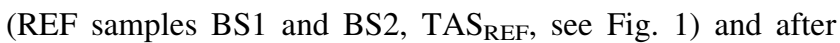

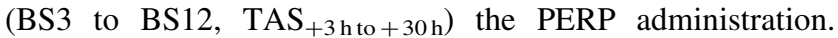
Then, iAUC were calculated, for each period of $3 \mathrm{~h}$, using the linear trapezoidal rule as follows:

1. $\mathrm{AUC}_{0 \text { to } 3 \mathrm{~h}}=\mathrm{TAS}_{+3 \mathrm{~h}}-\mathrm{TAS}_{\mathrm{REF}}$

2. $\mathrm{iAUC}_{0 \text { to } 6 \mathrm{~h}}=\mathrm{AUC}_{0 \text { to } 3 \mathrm{~h}}+\left(\mathrm{TAS}_{+6 \mathrm{~h}}-\mathrm{TAS}_{\mathrm{REF}}\right)$

3. $\mathrm{iAUC}_{0 \mathrm{htonh}}=\mathrm{AUC}_{0 \mathrm{hton}-3 \mathrm{~h}}+\left(\mathrm{TAS}_{\mathrm{nh}}-\mathrm{TAS}_{\mathrm{REF}}\right)$

For each time of plasma sample analysis, means of the five groups were compared using Student's $t$ test $^{31}$.

\section{Results}

Throughout the experimental period, mean daily intake of sheep was 755 (SEM 99) g/d for hay and 343 (SEM 24) g/d for concentrate. The administration of PERP did not modify feed intake.

\section{Lipaemia evolution in response to linseed oil infusion}

The evolution of lipid concentration in plasma, i.e. TAG, PL, FC, CE and NEFA over the first 3 weeks of linseed oil infusion (Phase 1) is shown in Table 2. Before infusion, total lipids (sum of TAG, PL, FC, CE and NEFA) were distributed as follows: $8.2 \%$ TAG; $41.8 \% \mathrm{PL} ; 0.6 \% \mathrm{FC} ; 48.7 \% \mathrm{CE}$; $0.8 \%$ NEFA. After $23 \mathrm{~d}$ of linseed oil infusion, plasma exhibited an overall hyperlipaemia ( $+40 \%$ total lipids), which was particularly due to the increase of the major lipids, i.e. PL and $\mathrm{CE}(+43 \%, P<0.05)$. In addition to the modifications of the lipid profile, the kinetics of evolution over the 3-week period differed between lipid classes. Indeed, TAG, FC and NEFA were exclusively increased during the first week of infusion $(+53 \%, P<0.05 ;+173 \%, \mathrm{NS} ;+67 \%, P<0.05$ respectively) and then decreased during the last 2 weeks, whereas PL and CE concentrations were continuously enhanced until the end of the infusion period.
Total antioxidant status evolution during the $30 \mathrm{~h}$ following plant extracts rich in polyphenol administration

The evolution of TAS during the $30 \mathrm{~h}$ following PERP or chopped hay (control) administration is reported in Fig. 2. The plasma of sheep given the PERP exhibited an overall increase of TAS (mean value for the four experimental groups at $+30 \mathrm{~h}:+134$ iAUC) whereas, in control, TAS decreased over the $30 \mathrm{~h}$ period $(-72 \mathrm{iAUC})$. The effect of PERP on TAS evolution from $+3 \mathrm{~h}$ to $+30 \mathrm{~h}$ was particularly pronounced with GP (+341 iAUC) while RO and CI both exhibited a rise of 81 iAUC and MA only increased TAS by 35 iAUC. Moreover, GP was the only PERP to significantly modify TAS $(P<0.05)$ in comparison with control.

Kinetics of TAS evolution differed with the PERP given. Indeed, in the GP group, TAS raised regularly from the beginning $(+3 \mathrm{~h})$ to the end $(+30 \mathrm{~h})$ of the blood collection period and the differences with the control group were significant $(P<0.05)$ from $15 \mathrm{~h}$ until $30 \mathrm{~h}$ after GP administration in rumen of sheep. In contrast, in RO, CI and MA groups, TAS was maximal between 12 and $21 \mathrm{~h}$ after PERP administration and it tended to decrease from $21 \mathrm{~h}$ to $30 \mathrm{~h}$.

\section{Recovery of polyphenols in plasma after the plant extracts rich in polyphenol administration}

Polyphenol patterns (Figs. 3 and 4) were determined in plasma collected between 12 and $21 \mathrm{~h}$, which corresponded to the period during which TAS reached the maximal value for most experimental groups (Fig. 2).

All the PERP tested led to the appearance of new polyphenols in comparison with control (Figs. 3(b) and 4(b)). With RO administration (Fig. 3(c)), five phenolic compounds were detected. One was eluted very early (at $9.0 \mathrm{~min}$, unknown A) and exhibited a high signal on the electrode set at $120 \mathrm{mV}$. Four other small signals were recorded on the electrode set at $360 \mathrm{mV}$ at $10.0 \mathrm{~min}$ (unknown B), $14.2 \mathrm{~min}$ (unknown C), 29.6 min (unknown D) and 31.6 min (unknown E). Despite the use of a large panel of standards (including rosmarinic acid), none of the five new phenolic molecules recovered in plasma of the RO group has been identified.

Table 2. Effect of duodenal linseed oil infusion (4\% DM ingested) for 9,16 and $23 \mathrm{~d}$ in castrated sheep ( $n$ 5) on plasma concentrations (mg/dl) of TAG, phospholipid, free cholesterol, cholesteryl ester and NEFA*

(Mean values with their standard errors)

\begin{tabular}{|c|c|c|c|c|c|c|c|c|c|}
\hline & \multicolumn{8}{|c|}{ Time after the beginning of linseed oil infusion (d) } & \multirow[b]{3}{*}{$P^{\star}$} \\
\hline & \multicolumn{2}{|c|}{0} & \multicolumn{2}{|c|}{9} & \multicolumn{2}{|c|}{16} & \multicolumn{2}{|c|}{23} & \\
\hline & Mean & SE & Mean & SE & Mean & SE & Mean & SE & \\
\hline TAG & $10 \cdot 7^{\mathrm{a}}$ & 0.9 & $16 \cdot 5^{\mathrm{b}}$ & $2 \cdot 9$ & $12 \cdot 4^{\mathrm{ab}}$ & $2 \cdot 0$ & $9 \cdot 6^{a}$ & $1 \cdot 1$ & 0.05 \\
\hline Phospholipid & $55 \cdot 0^{\mathrm{a}}$ & 3.3 & $68 \cdot 0^{\mathrm{ab}}$ & $5 \cdot 8$ & $70 \cdot 2^{a b}$ & $7 \cdot 2$ & $78 \cdot 9^{b}$ & $10 \cdot 3$ & 0.10 \\
\hline Free cholesterol & 0.8 & 0.2 & $2 \cdot 2$ & 0.6 & 1.4 & 0.2 & 1.4 & 0.7 & 0.17 \\
\hline Cholesteryl ester & $64 \cdot 0^{\mathrm{a}}$ & $3 \cdot 8$ & $74 \cdot 6^{\mathrm{ab}}$ & $6 \cdot 9$ & $79 \cdot 5^{a b}$ & $8 \cdot 0$ & $91 \cdot 8^{b}$ & $10 \cdot 5$ & 0.05 \\
\hline NEFA & $1.0^{\mathrm{a}}$ & 0.2 & $1.7^{\mathrm{b}}$ & 0.1 & $1 \cdot 7^{\mathrm{b}}$ & $0 \cdot 1$ & $1.5^{\mathrm{b}}$ & 0.2 & 0.01 \\
\hline
\end{tabular}

Data were subjected to general one-way ANOVA with time as the main factor. When the effect was declared as a trend $(P \leq 0.1)$ or significant $(P \leq 0.05)$, means of the four times were compared using Student's $t$ test. Mean values within a row with unlike superscript letters were statistically different $(P<0.05)$.

${ }^{*}$ For details of diets and procedures, see Materials and methods. 

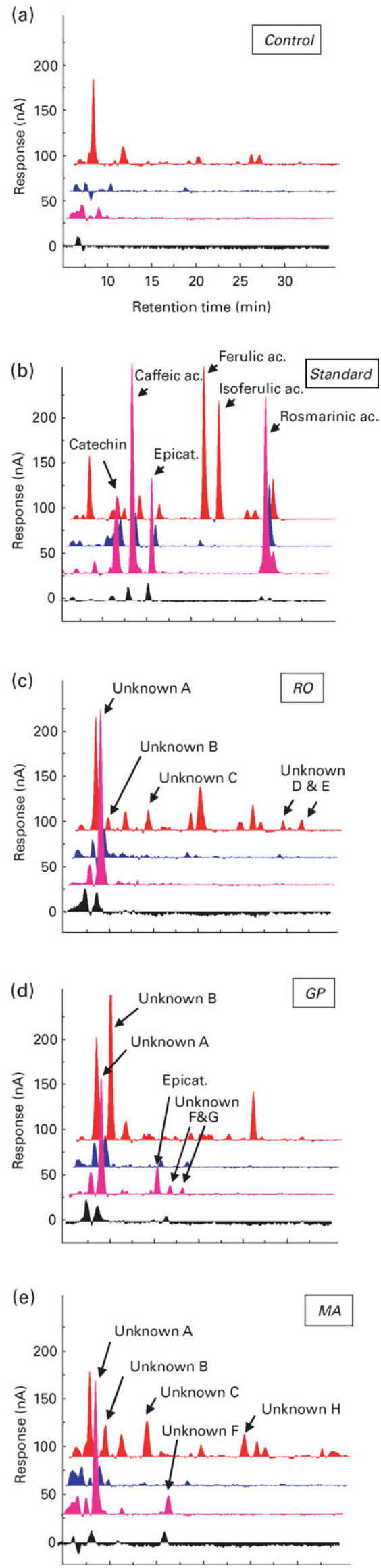

After GP administration (Fig. 3(d)), among the five signals recorded, the first two peaks detected had the same retention times (9.0 and 10.0 $\mathrm{min}$ ) and were detected on the same electrodes (set at 120 and $360 \mathrm{mV}$ ) as the first two peaks recorded in RO plasma (unknown A and B). Moreover, three other signals were recorded on the electrode set at $120 \mathrm{mV}$ at $15 \cdot 3,16 \cdot 9$ (unknown F) and 19.4 min (unknown G). Among these six phenolic molecules detected, the fourth eluted (at $15.3 \mathrm{~min}$ ) was identified as epicatechin.

With MA treatment (Fig. 3(e)), five new phenolic molecules appeared in plasma. The first two (recorded at 9.0 and $10.0 \mathrm{~min}$ ) had the same chromatographic and electrochemical behaviours as the first molecules detected with both RO and GP extracts (unknown A and B). The third and the fourth molecules detected had the same chromatographic and electrochemical behaviours as the unknown $\mathrm{C}$ from RO plasma and the unknown $\mathrm{F}$ from GP plasma. Finally, a fifth signal was recorded at $24.9 \mathrm{~min}$. Despite the use of many standards of flavonoids and phenolic acids, none of the five molecules detected has been identified.

Finally, with CI (Fig. 4(c)), a unique signal with a high intensity was recorded at $40.0 \mathrm{~min}$ simultaneously on the electrodes set at 600,720 and $840 \mathrm{mV}$. This molecule was identified as naringenin.

\section{Effect of plant extracts rich in polyphenols on lipoperoxidation in plasma}

The effect of PERP on lipoperoxidation was evaluated ex vivo by measuring the kinetics of CD generation in pooled plasma collected before and after the administration of PERP in the rumen. Lag phase and oxidation rate, which respectively represent the length of the resistance phase and the speed of the propagation phase of lipoperoxidation, are reported in Table 3.

All the PERP tested increased the length of the lag phase when compared with the control group $(+5.9 \mathrm{~min}, P<0.02)$. This rise of the resistance phase was particularly pronounced with MA and RO, which respectively increased the length of lag phase by $78 \%(P<0.01)$ and $69 \%(P=0.01)$, while $\mathrm{CI}$ and GP increased lag phase by $46 \%(P=0.03)$ and $29 \%$ $(P=0 \cdot 05)$ respectively.

The speed of the propagation phase, as represented by the oxidation rate, was reduced with the four PERP tested $\left(-1.7 \mathrm{~A}_{234} / \mathrm{min}, P<0.01\right)$ in comparison with the control. This parameter was particularly reduced with MA $(-57 \%$, $P<0.01)$ while RO and GP both reduced the oxidation rate by $16 \%(P<0 \cdot 01)$ and $\mathrm{CI}$ extracts by $14 \%(P<0 \cdot 01)$.

\section{Discussion}

The present experiment was conducted to investigate the bioavailability and the antioxidant capacity of four PERP in ruminants. Considering that most studies in this field of

Fig. 3. HPLC chromatograms with multi-electrode coulometric detection of aglycone polyphenols from (a) chopped hay (control); (b) plasma in which standards were added or from pooled plasma of sheep given, directly in the rumen, as a single acute dose (10\% DM ingested); (c) rosemary (RO); (d) grape (GP); (e) marigold (MA). Electrode potentials were set at $0 \mathrm{mV}$ (black), $120 \mathrm{mV}$ (pink), $240 \mathrm{mV}$ (dark blue), $360 \mathrm{mV}$ (red), $480 \mathrm{mV}$ (light green), $600 \mathrm{mV}$ (yellow), $720 \mathrm{mV}$ (dark green) and $840 \mathrm{mV}$ (light blue) and only the electrodes on which signals were recorded are represented. For details of plasma pooling and analysis procedures, see Materials and methods section. 

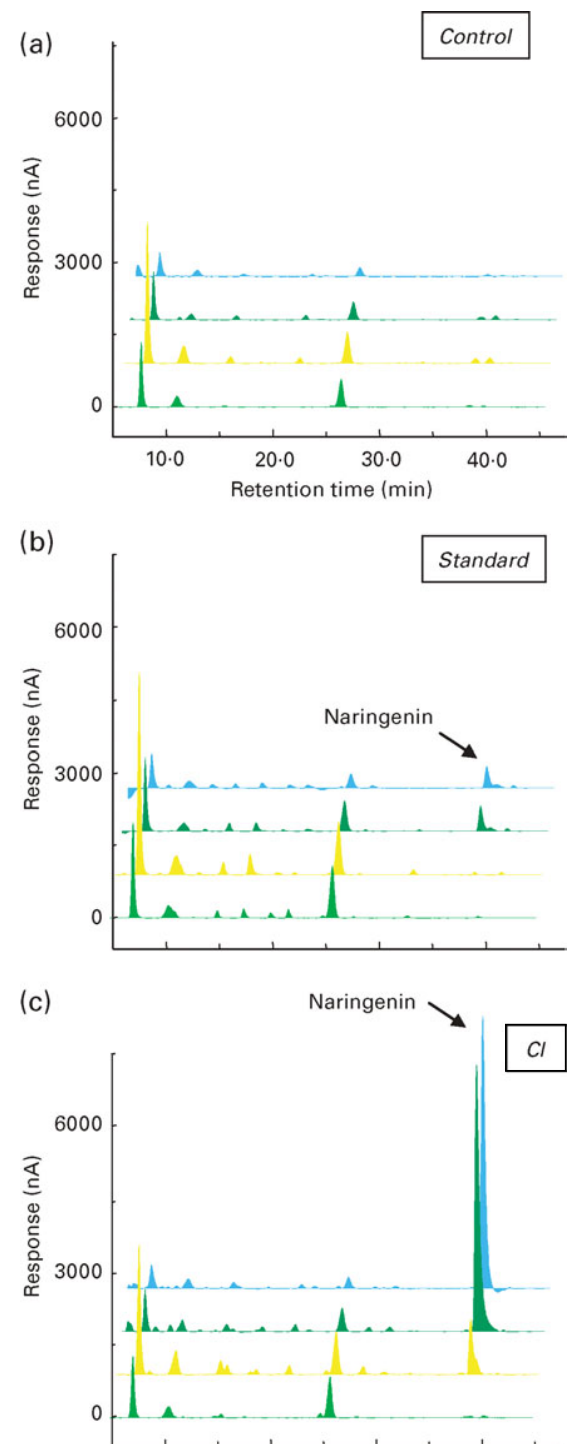

Fig. 4. HPLC chromatograms with multi-electrode coulometric detection of aglycone polyphenols from (a) chopped hay (control), (b) plasma in which standards were added or from pooled plasma of sheep given, directly in the rumen, as a single acute dose (10\% DM ingested) or (c) citrus (Cl). Electrode potentials were set at $0 \mathrm{mV}$ (black), $120 \mathrm{mV}$ (pink), $240 \mathrm{mV}$ (dark blue), $360 \mathrm{mV}$ (red), $480 \mathrm{mV}$ (light green), $600 \mathrm{mV}$ (yellow), $720 \mathrm{mV}$ (dark green) and $840 \mathrm{mV}$ (light blue) and only the electrodes on which signals were recorded are represented. For details about plasma pooling and analysis procedures, see Materials and methods.

research have been performed in monogastric species, the relevance of the present results will be discussed by referring to data obtained from monogastric studies.

To make plasma highly susceptible to lipoperoxidation, linseed oil (4\% DM) was continuously infused in the duodenum of sheep. After 3 weeks of treatment, plasma of the sheep was characterized by hyperphospholipaemia as well as hypercholesterolaemia. Similar experiments were previously performed in the laboratory on steers and led to the same modifications of lipid profile $(+63 \% \mathrm{PL} \text { and }+100 \% \mathrm{CE})^{9}$. The alteration of the lipid profile was associated with a six-fold increase of linolenic acid (18:3n-3) concentration in plasma and significant reduction of lag phase $(-40 \%)$, suggesting a higher susceptibility of plasma lipids to lipoperoxidation ${ }^{9}$.
In vivo antioxidant capacity of plant extracts rich in polyphenols

The addition of PERP in the rumen of sheep, and more especially of GP, increased the TAS of plasma, whereas the plasma TAS of sheep given chopped hay (control) regularly decreased during the $30 \mathrm{~h}$ period. This is not consistent with a recent experiment ${ }^{32}$ conducted in Charolais bulls given RO extract, but the low level of extract administered ( $0.02 \%$ DM ingested) as well as the time of plasma collection ( $3 \mathrm{~h}$ after the extract administration) were probably not appropriate to induce a significant evolution of TAS in the plasma of bulls. In the present experiment, we deliberately chose to test a supranutritional dose (10\% DM ingested) but further investigations should be performed to determine which dose of our PERP should be recommended for a nutritional treatment. In rodents, we have previously shown that PERP (RO, GP, CI and MA) given at a nutritional dose $(0.5 \%$ DM) did not increase TAS in plasma collected in the postabsorptive state $^{22}$. In the same experiment, the level of malondialdehyde (a final metabolite of lipoperoxidation) was nevertheless reduced, suggesting that the PERP were bioefficient antioxidants and it has been concluded that the level of polyphenols (or their metabolites) in plasma was too low to increase TAS. A human study showed that a unique acute dose (240-300 g) of various polyphenol-rich foods significantly increased TAS $(+7-25 \%)$ during the 4-h period following consumption ${ }^{33}$. Then, the dose of PERP rather than the time of blood collection may be the limiting parameter to modify TAS in plasma.

Among the four PERP tested, GP was by far the most efficient PERP to improve TAS in sheep plasma. This difference could have been expected since GP had the greatest content in polyphenols but, on the basis on a previous study conducted in rats $^{22}$, it was assumed that polymeric proanthocyanidins (the main polyphenol in our GP extract) were not bioavailable. The present experiment then demonstrated that, contrary to monogastrics, ruminants can make polymeric proanthocyanidins bioavailable and benefit from their strong antioxidant capacity. This may involve a hydrolysis of proanthocyanidin polymers into bioavailable and bioactive monomers (catechin or epicatechin) by hydrolysed rumen micro-organisms, since it has been shown ${ }^{34}$ that polymeric proanthocyanidins are poorly recovered after their transit through the rumen.

The kinetics of TAS evolution were different between the four PERP. Indeed, with RO, CI and MA the highest value of TAS was reached between 12 and $21 \mathrm{~h}$, whereas with GP, TAS kept on rising for $30 \mathrm{~h}$. These differences might be due to the high level of proanthocyanidins in GP, which can form stable complexes with proteins ${ }^{35}$ and bind to the plant particles constituting the solid phase in the rumen ${ }^{36}$. In contrast, the other PERP might have been rapidly transferred, together with the liquid phase, towards the small intestine.

\section{Recovery of polyphenols in plasma}

The administration of the four PERP induced marked changes in the polyphenol patterns in sheep plasma. Moreover, the present experiment revealed that polyphenols were still present in sheep plasma more than $10 \mathrm{~h}$ after administration. Until now, 
Table 3. Evolution* of lag phase and oxidation rate in plasma of sheep given one of the four plant extracts rich in polyphenols (PERP) as a single acute dose (10\% DM ingested) directly in the rument

(Mean values with their standard errors for five animals)

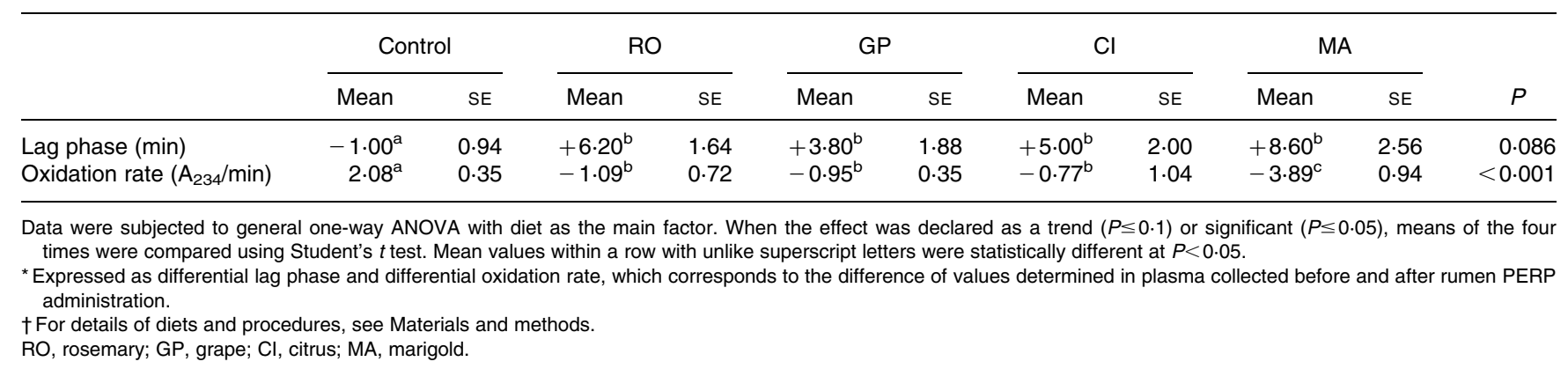

bioavailability of polyphenols in ruminants has been poorly documented and only one experiment, conducted in dairy cows and goats, reported the presence of isoflavones in milk, suggesting that polyphenols could be absorbed in ruminants $^{37}$.

RO contains mainly rosmarinic acid as well as some diterpene phenolics ${ }^{22}$. In monogastrics, it has been shown ${ }^{38,39}$ that rosmarinic acid is mostly recovered intact in plasma within $30 \mathrm{~min}$ and its metabolites (i.e. ferulic, caffeic and coumaric acids) appear within $8 \mathrm{~h}$. The sheep plasma collected between 12 and $21 \mathrm{~h}$ after RO administration did not contain rosmarinic acid or its metabolites but other phenolic compounds were recovered. Two hypotheses could explain these contrasting results between monogastrics and ruminants. First, it is possible that rosmarinic acid and its metabolites were absorbed in sheep but were already excreted when we collected the plasma. Second, the presence of unknown polyphenols in sheep plasma may suggest that rosmarinic acid was metabolized in rumen and the anaerobic microbial activity of the rumen may explain the differences with monogastrics. Concerning the phenolic diterpenes, they could not be identified with the standard used in the present experiment but their presence cannot be excluded.

Proanthocyanidins (or condensed tannins) are the most abundant phenolic compounds in GP. They are oligomeric or polymeric flavanols composed of catechin and epicatechin. The GP extract used in the present experiment contained more than $80 \%$ proanthocyanidins, which were mostly under a polymeric form $(>70 \%)$. The administration of GP directly in the rumen of sheep led to the appearance of five different phenolic compounds in plasma, among which epicatechin was identified. The absorption and the metabolism of polymeric proanthocyanidins have been extensively studied in monogastrics. It has been reported ${ }^{40}$ that polymeric proanthocyanidins are catabolized into phenolic acids of low molecular weight by human colonic microflora. Other authors have suggested that polymeric proanthocyanidins are hydrolysed by gastric fluids to lower molecular compounds such as catechin and epicatechin ${ }^{41,42}$ but it was not confirmed in a recent study $^{43}$ showing that procyanidins are stable during gastric transit in human subjects. Finally, it was reported ${ }^{44,45}$ that polymeric proanthocyanidins are not decomposed into bioavailable monomers in the monogastric digestive system and it was concluded that only the monomeric components of GP (catechin and epicatechin) are absorbed in monogastrics.
Considering that our GP extract contained a minor proportion of monomeric components, it is likely that the relatively high level of epicatechin recovered in the plasma of sheep came from the biodegradation of polymeric proanthocyanidins by rumen micro-organisms. This would suggest that, contrary to monogastrics, ruminants can benefit from the strong antioxidant properties of polymeric proanthocyanidins by metabolizing them into bioavailable compounds with intact flavonoid-ring structure (epicatechin).

The CI extract given to sheep in the present experiment contained more than $95 \%$ naringin (naringenin-7-rhamnoglucoside), which is a glycosylated flavanone specific to citrus fruits $^{46}$. In monogastrics, the glycosylated form cannot be absorbed by the small intestine and naringin is transferred to the large intestine, where the colonic microflora hydrolyse the glycosidic fraction and release the aglycone (naringenin), which is then absorbed ${ }^{47}$. In the present experiment, naringenin was recovered in a large proportion in the plasma of sheep and no other metabolite was detected. This suggests that ruminal metabolism did not modify the flavanone structure of naringin and that, as in monogastrics, the hydrolysis of the glycosidic fraction was required before absorption. However, in contrast with monogastrics, it is likely that the hydrolysis of the glycosidic fraction occurred in the rumen rather than in the large intestine. Indeed, the delays of naringenin absorption were relatively similar in monogastrics ${ }^{47}$ and in our sheep (around $10 \mathrm{~h}$ after administration), although it should have been much longer in sheep due to the rumen retention of the CI extract. However, further experiments using ruminal fluid should be performed to confirm that rumen micro-organisms can hydrolyse the glycosidic fraction of naringin and release the aglycone form without metabolizing it further.

MA contains many antioxidant compounds, including phenolic acids such as vanillic, caffeic, chlorogenic, p-coumaric and ferulic acids ${ }^{25}$. We detected five polyphenols in the plasma of sheep given MA but none of them was identified as the phenolic acids listed earlier, suggesting that these molecules were further metabolized in the rumen. However, feeding trials with MA extract have never been reported in literature and no data on the circulating polyphenols are available.

Finally, the analysis of polyphenol patterns in plasma of sheep given RO, GP and MA revealed the presence of common phenolic compounds, among which the unknown 
compound A (first molecule eluted) represented a major polyphenol, exhibiting a high polarity, a low molecular weight and a high reducing capacity (according to its response at low potentials). This common metabolite could not be identified in the present experiment but further analysis should be performed since this phenolic compound may be the final metabolite of many polyphenols in sheep and it could play a crucial antioxidant function in sheep plasma.

\section{Bioefficiency of plant extracts rich in polyphenols to limit lipoperoxidation}

The four PERP tested reduced the susceptibility of plasma to lipoperoxidation since lag phase (a parameter related to the resistance of lipids to peroxidation) was significantly increased after PERP administration in the rumen. This effect was particularly pronounced with MA, which is rather surprising since MA did not exhibit the strongest reducing capacity in vitro and was not associated with a significant increase of TAS in plasma of sheep. In contrast, GP was the most efficient in vitro and significantly improved TAS but was not more bioefficient than the other PERP to increase lag phase. Since we previously demonstrated that GP's polyphenols (or their metabolites) were relatively well absorbed (see Polyphenol patterns), it is likely that the chemical structure of the bioactive compounds from GP was not appropriate (e.g. too hydrophile) to prevent lipid oxidation. In our previous experiment conducted in rats ${ }^{22}$, neither MA nor GP significantly increased lag phase in plasma, and RO was the only bioefficient extract concerning this parameter. This conclusively shows that the bioefficiency of PERP to prevent lipoperoxidation cannot be predicted from in vitro measurement. Moreover, the differences observed between sheep and rats provided further evidence that rumen metabolism of PERP generated specific polyphenol metabolites with different biological properties than the metabolites present in rat plasma.

The ability of PERP to protect lipids from peroxidation also suggests that the polyphenols or their metabolites were integrated into the lipid structure or at the aqueous-lipid interface. This would suggest that, contrary to the majority of polyphenols, those brought by our PERP and recovered in the plasma of sheep were not bound to proteins ${ }^{17}$ but were either localized at the aqueous-lipid interface as previously reported for (-)-epigallocatechin-3-gallate ${ }^{48}$ or incorporated in lipoproteins, as was described with genistein ${ }^{49}$. Moreover, the stronger effect of MA may be due to the presence of carotenoids in addition to polyphenols ${ }^{50}$.

The four PERP tested, and more especially MA, reduced the oxidation rate, a parameter related to the propagation phase of lipoperoxidation. This effect was not observed in rats ${ }^{22}$, confirming that polyphenol metabolites generated in ruminants were different from the ones recovered in the plasma of rats. In ruminants, PERP were efficient to prevent both the initiation and the propagation of lipoperoxidation, suggesting their ability to quench both the reactive oxygen species (or any other initiator) and the lipoperoxide generated. This biological property, usually attributed to vitamin $\mathrm{E}^{51}$, confirms that the polyphenols were integrated into the lipid structure or at the aqueous-lipid interface, since they would not have been able to quench lipoperoxides by being linked to proteins.
In conclusion, the present experiment showed that the four PERP tested kept their antioxidant capacity in vivo in ruminants. Moreover, in the case of GP, the action of rumen micro-organisms was beneficial, since they improved the bioavailability of polymeric proanthocyanidins. Rumen also has a beneficial effects by delaying the absorption of GP polyphenols, leading to a longer time of action than the other PERP, which were more rapidly transferred into the plasma of sheep. The four PERP tested in the present experiment were also particularly efficient to limit plasma susceptibility to lipoperoxidation and should therefore represent a specific interest in ruminants fed $n$-3 PUFA-rich diets. The MA extract was the most bioefficient, probably because of the lipophilicity of its bioactive compounds.

The present work was an essential step to investigate the interest of polyphenols as new antioxidants in animal nutrition and their essential role to improve growth performances and product quality. The encouraging results obtained concerning the biological activity of the four PERP selected lead us to envisage further work to elucidate the nature and the structure of the active metabolites found in plasma and to better understand the metabolic pathways of polyphenols in ruminants. Moreover, a supranutritional dose was deliberately used in the present experiment but a dose-response study should be conducted in order to determine the optimum level of administration.

\section{Acknowledgements}

This experiment was financially supported by the European Union (via a EUREKA research programme). We thank our industrial partner Phytosynthese (Saint-Bonnet de Rochefort, France) for supplying PERP. We also wish to address specific thanks to Mrs Anne-Sophie Bage, Françoise Duboisset, Christiane Legay, Marinett Martinaud and Agnès Thomas for their technical assistance as well as to Mr Daniel Thomas, Sébastien Alcouffe and Miche Fabre for the animal care.

\section{References}

1. Aurousseau B (2002) Oxygen radicals in Farm animals. Physiological effects and consequences on animal products. INRA Prod Anim 15, 67-82.

2. Moller P, Wallin H \& Knudsen LE (1996) Oxidative stress associated with exercise, psychological stress and life-style factors. Chem Biol Inter 102, 17-36.

3. Miller JK \& Brzezinska-Slebodzinska E (1993) Oxidative stress, antioxidants and animal function. J Dairy Sci 76, 2812-2823.

4. Takahata K, Monobe K, Tada M \& Weber P (1998) The benefits and risks of n-3 polyunsaturated fatty acids. Biosci Biotechnol Biochem 62, 2079-2085.

5. Clinquart A, Micol D, Brundseaux C, Dufrasne I \& Istasse L (1995) Use of lipid supplement in fattening bovines. INRA Prod Anim 8, 29-42.

6. Wood JD \& Enser M (1997) Factors influencing fatty acids in meat and the role of antioxidants in improving meat quality. Br J Nutr 78, S49-S60.

7. Bauchart D, Gladine C, Gruffat D, Leloutre L \& Durand D (2005) Effects of diets supplemented with oil seeds and vitamin $\mathrm{E}$ on specific fatty acids of rectus abdominis muscle in Charolais fattening bulls. In Indicators of Milk and Beef Quality, 1st 
ed., pp. 431-436 [JF Hocquette and S Gigli, editors]. Wageningen The Netherlands: Wageningen Academic Publishers.

8. Frémont L, Gozzélino MT, Franchi MP \& Linard A (1998) Dietary flavonoids reduce lipid peroxidtion in rats fed polyunsaturated or monounsaturated fat diets. J Nutr 128, 1495-1502.

9. Scislowski V, Bauchart D, Gruffat D, Laplaud PM \& Durand D (2005) Effect of dietary n-6 or n-3 polyunsaturated fatty acids protected or not against ruminal hydrogenation on plasma lipids and their susceptibility to peroxidation in fattening steers. J Anim Sci 83, 2162-2174.

10. Allard J, Kurian R, Aghdassi E, Muggli R \& Royall D (1997) Lipid peroxidation during n-3 fatty acid and vitamin E supplementation in humans. Lipids 32, 535-541.

11. Miret S, Saiz MP \& Mitjavila MT (2003) Effect of fish oil- and olive oil-rich diets on iron metabolism and oxidative stress in rat. Br J Nutr 89, 11-18.

12. Azzi A \& Stocker A (2000) Vitamin E: non-antioxidant roles. Prog Lipid Res 39, 231-255.

13. Mukai K, Sawada K, Kohno Y \& Terao J (1993) Kinetic study of the prooxidant effect of tocopherol. Hydrogen abstraction from lipid hydroperoxides by tocopheroxyls in solution. Lipids 28, 747-752.

14. Bravo L (1998) Polyphenols: chemistry, dietary sources, metabolism, and nutritional significance. Nutr Rev 56, 317-333.

15. Andersen ML, Lauridsen RK \& Skibsted LH (2003) Optimising the use of phenolic compounds in food. In Phytochemical Functional Foods, 1st ed., pp. 315-346 [I Johnson and G Williamson, editors]. Cambridge, England: Woodhead Publishing Limited.

16. Grün IU, Ahn J, Andrew DC \& Lorenzen CL (2006) Reducing oxidation of meat. Food Technol 37-43.

17. Manach C, Scalbert A, Morand C, Rémézy C \& Jimenez L (2004) Polyphenols: food sources and bioavailability. Am J Clin Nutr 79, 727-747.

18. Papageorgiou G, Botsoglou N, Govaris A, Giannenas I, Iliadis S \& Botsoglou E (2003) Effect of dietary oregano oil and \&-tocopheryl acetate supplementation on iron-induced lipid oxidation of turkey breast, thigh, liver and heart tissues. J Anim Physiol Anim Nutr 87, 324-335.

19. Young JF, Stagsted J, Jensen SK, Karlsson AH \& Henckel P (2003) Ascorbic acid, $\alpha$-tocopherol and oregano supplements reduce stress-induced deterioration of chicken meat quality. Poultry Sci 82, 1343-1351.

20. Giannenas IA, Florou-Paneri P, Botsoglou NA, Christaki E \& Spais AB (2005) Effect of supplementing feed with oregano and/or $\alpha$-tocopheryl acetate on growth of broiler chickens and oxidative stability of meat. J Anim Feed Sci 14, 521-535.

21. Jouany JP (1994) Fermentations in rumen and their improvement. INRA Prod Anim 7, 207-225.

22. Gladine C, Morand C, Rock E, Bauchart D \& Durand D (In the press) Plant extracts rich in polyphenols (PERP) are efficient to prevent lipoperoxidation in plasma lipids from animals fed n-3 PUFA supplemented diets. Anim. Feed Sci. Technol.

23. Gladine C, Morand C, Rock E, Gruffat D, Bauchart D \& Durand $\mathrm{D}$ (In the press) The antioxidative effect of plant extracts rich in polyphenols differs between liver and muscle tissues in rats fed n-3 PUFA rich diets. Anim. Feed Sci. Technol.

24. Del Baño MJ, Lorente J, Castillo J, Benavente-Garcia OO, Del Rio JA, Ortuño A, Quirin KW \& Gerard D (2003) Phenolic diterpenes, flavones and rosmarinic acid distribution during the development of leaves, flowers, stems, and roots of Rosmarinus officinalis. Antioxidant activity. J Agric Food Chem 51, 4247-4253.

25. Matysik G, Wojciak-Kosior M \& Paduch R (2005) The influence of Calendulae officinalis flos extracts on cell cultures and the chromatographic analysis of extracts. $J$ Pharm Biomed Anal 38, 285-292.
26. Leplaix-Charlat L, Bauchart D, Durand D, Laplaud PM \& Chapman MJ (1996) Plasma lipoproteins in preruminant calves fed diets containing tallow or soybean oil with and without cholesterol. J Dairy Sci 79, 1267-1277.

27. Morand C, Manach C, Donovan J \& Rémézy C (2001) Preparation and characterization of flavonoid metabolites present in biological samples. Methods Enzymol 335, 115-121.

28. Miller NJ, Rice-Evans C, Davies MJ, Gopinathan V \& Milner A (1993) A novel method for measuring antioxidant capacity and its application to monitoring the antioxidant status in premature neonates. Clin Sci (Lond) 84, 407-412.

29. Schnitzer E, Pinchuk M, Fainaru M, Schafer Z \& Lichtenberg D (1995) Copper-induced lipid oxidation in unfractionated plasma: the lag preceding oxidation as a measure of oxidation resistance. Biochem Biophys Res Commun 216, 854-861.

30. Esterbauer H, Striegl G, Puhl H \& Rotheneder M (1989) Continuous monitoring of in vitro oxidation of human low density lipoprotein. Free Radic Res Commun 6, 67-75.

31. Snedecor GW \& Cochran WG (1979) The comparison of two samples. In Statistical Methods, 6th edn. Ames: The Iowa State University Press.

32. O'Grady MN, Maher M, Troy DJ, Moloney AP \& Kerry JP (2006) An assessment of dietary supplementation with tea catechins and rosemary extract on the quality of fresh beef. Meat Sci 73, $132-143$.

33. Cao G, Russell RM, Lischner N \& Prior RL (1998) Serum antioxidant capacity is increased by consumption of strawberries, spinach, red wine or vitamin C in elderly women. $J$ Nutr $\mathbf{1 2 8}$, 2383-2390.

34. Terrill TH, Waghorn GC, Woolley DJ, Nabb WC \& Barry TN (1994) Assay and digestion of 14C-labelled condensed tannins in the gastrointestinal tract of sheep. Br J Nutr 72, 467-477.

35. Singh B, Bhat TK \& Singh B (2003) Potential therapeutic applications of some antinutritional plant secondary metabolites. J Agric Food Chem 51, 5579-5597.

36. McAllister TA, Bae HD, Jones GA \& Cheng KJ (1994) Microbial attachment and feed digestion in the rumen. $J$ Anim Sci 72, 3004-3018.

37. Sakakibara H, Viala D, Doreau M \& Besle JM (2003) Clover isoflavones move to cow's milk. In 1st International Conference on Polyphenols and Health (Poster). Vichy, France.

38. Baba S, Osakabe N, Natsume M, Yasuda A, Muto Y, Hiyoshi K, Takano H, Yoshikawa T \& Terao J (2005) Absorption, metabolism, degradation and urinary excretion of rosmarinic acid after intake of Perilla frutescens extract in humans. Eur $J$ Nutr 44, 1-9.

39. Konishi Y, Hitomi Y, Yoshida M \& Yoshioka E (2005) Pharmacokinetics study of caffeic and rosmarinic acids in rats after oral administration. J Agric Food Chem 53, 4740-4746.

40. Déprez S, Brezillon C, Rabot S, Philippe C, Mila I, Lapierre C \& Scalbert A (2000) Polymeric proanthocyanidins are catabolized by human colonic microflora into low-molecular-weight phenolic acids. $J$ Nutr 130, 2733-2738.

41. Spencer JPE, Chaudry F, Pannala AS, Srai SK, Debnam A \& Rice-Evans C (2000) Decomposition of cocoa procyanidins in the gastric milieu. Biochem Biophys Res Com 272, 236-241.

42. Spencer JPE, Schroeter H, Shenoy B, Srai SKS, Debnam ES \& Rice-Evans C (2001) Epicatechin is the primary bioavailable form of the procyanidin dimmers B2 and B5 after transfer across the small intestine. Biochem Biophys Res Com 285, 588-593.

43. Rios LY, Bennett RN, Lazarus SA, Rémézy C, Scalbert A \& Williamson $\mathrm{G}$ (2002) Cocoa procyanidins are stable during gastric transit in humans. Am J Clin Nutr 76, 1106-1110.

44. Donovan JL, Manach C, Rios L, Morand C, Scalbert A \& Rémézy C (2002) Procyanidins are not bioavailable in rats fed 
a single meal containing a grapeseed extract or the procyanidin dimmer $\mathrm{B}_{3}$. Br J Nutr 87, 299-306.

45. Nakamura Y \& Tonogai Y (2003) Metabolism of grape seed polyphenols in the rat. J Agric Food Chem 51, 7215-7225.

46. Ortuño A, Garcia-Puig D, Fuster MD, Pérez ML, Sabater F, Porras I, Garcia-Lidon A \& Del Rio JA (1995) Flavanone and nootkatone levels in different varieties of grapefruit and pummelo. J Agric Food Chem 43, 1-5.

47. Felgines C, Texier O, Morand C, Manach C, Scalbert A, Régerat F \& Rémézy C (2000) Bioavailability of the flavanone naringenin and its glycosides in rats. Am J Physiol Gastrointest Liver Physiol 279, G1148-G1154.

48. Aldini G, Yeum K-J, Carini M, Krinsky NI \& Russell RM (2003) (-)-Epigallocatechin-(3)-gallate prevents oxidative damage in both the aqueous and lipid compartments of human plasma. Biochem Biophys Res Com 302, 409-414.

49. Kaamanen M, Adlercreutz H, Jauhiainen M \& Tikkanen MJ (2003) Accumulation of genistein and lipophilic genistein derivatives in lipoproteins during incubation with human plasma in vitro. Biochim Biophys Acta 1631, 147-152.

50. Hadden WL, Watkins RH, Levy LW, Regalado E, Rivadeneira DM, van Breemen RB \& Schwartz SJ (1999) Carotenoid composition of Marigold (Tagetes erecta) flower extract used as nutritional supplement. J Agric Food Chem 47, 4189-4194.

51. Khalil A (2002) Mécanisme moléculaire de l'effet protecteur de la vitamine E dans l'athérosclérose. Can J Physiol Pharmacol 80, 662-669. 